\title{
Valorización de Berberis mikuna Job. una especie frutal nativa de Tucumán con potencial nutracéutico
}

\author{
N. Guastavino ${ }^{1,2}$ G. Dantur ${ }^{3}$, A. Ceribelli ${ }^{4}$, ME. Arena ${ }^{1,2}$ y S. Radice ${ }^{1,2}$ \\ 'Laboratorio de Fisiología Vegetal, FAyCA, Universidad de Morón, Machado 914, Morón (B1708E0H), Buenos Aires, Argentina. \\ ${ }^{2}$ CONICET. \\ ${ }^{3}$ Estudiante de Ciencias Biológicas. Facultad de Ciencias Naturales e Instituto Miguel Lillo, Tucumán. \\ 4Estudiante de Corso di Laurea in Scienze e Tecnologie Agrarie, Scuola di Agraria DISPAA. Universidad de Florencia Italia. \\ E-mail: siradice@yahoo.com
}

\section{Resumen}

Berberis mikuna es una especie nativa del NO argentino. El estudio del ciclo vegetativo y reproductivo de esta especie aportará el conocimiento necesario para la domesticación y la posibilidad de desarrollo de un programa de mejoramiento. Esta especie fue descrita por Job en 1942 como un árbol de $6 \mathrm{~m}$ de alto que crece entre los 1200 y 3000 m.s.n.m., por tal motivo, la zona de estudio seleccionada fue Alto de Medina (Tucumán) en donde se realizó una marcación de ejemplares que crecen en diversas situaciones agroclimáticas para estudiar su ciclo reproductivo. Si bien los resultados son muy preliminares podemos decir que las plantas mostraron marcadas diferencias en el ciclo de floración y fructificación. Así el 27 de octubre de 2017 se encontraron plantas con 90 $\%$ de capullos florales mientras que otras tenían $70 \%$ de frutos ya formados. Los frutos cosechados a principios de enero también fueron diferentes en sus propiedades físicas y químicas. Estos variaron entre 0,055 y 0,032 g de PS con diámetro promedios de 7,3 y 4,7 mm. El contenido de sólidos solubles, antocianinas y de fenoles totales medido en los frutos también fue significativamente diferente según la planta estudiada. El objetivo del presente trabajo será el de abordar un estudio integral de la especie para su posterior uso sustentable.

Palabras claves: pequeños frutos, biología reproductiva, frutos, antocianinas 